\title{
Distrofia Simpática Reflexa Pós Herpes Zoster ${ }^{(*)}$
}

\section{Reflex Sympathetic Dystrophy Following Herpes Zoster}

\author{
Catia Susana Harumi Minami ${ }^{(1)}$, Mônica Duarte Costa $^{(1)}$, Silvio Figueira Antônio ${ }^{(2)}$ e Wiliam Habib Chahade ${ }^{(3)}$
}

\section{RESUMO}

A distrofia simpática reflexa (DSR) é uma síndrome dolorosa pouco entendida que consiste na presença de múltiplos sinais e sintomas incluindo dor, edema, alterações cutâneas distróficas e disfunção autonômica envolvendo uma ou mais extremidades. Nem sempre a DSR pós-herpes zóster é facilmente reconhecida. Poucos relatos dessa complicação foram publicados, o que torna interessante esta descrição. Apresentamos o caso de uma mulher branca, de 65 anos de idade cujos sinais e sintomas característicos de DSR no membro superior direito surgiram após 4 semanas do aparecimento das lesões de herpes zóster envolvendo trajeto dermatomérico. Com o diagnóstico precoce e abordagem terapêutica com calcitonina nasal e fisioterapia houve melhoras funcional e álgica satisfatórias em um mês de tratamento.

Palavras-chave: distrofia simpática reflexa, algoneurodistrofia, herpes zóster.

\section{INTRODUÇÃO}

A distrofia simpática reflexa (DSR) é uma síndrome clínica caracterizada por dor intensa, alterações cutâneas distróficas, edema, rigidez e disfunção autonômica de uma ou mais extremidades $^{(1)}$. Apesar de ter sido descrita em 1864 por Mitchell, a sua patogênese ainda é pouco compreendida e trata-se de uma entidade clínica não muito reconhecida. A hipótese classicamente aceita é a de uma alteração do sistema nervoso simpático ${ }^{(2)}$. Participação de reação inflamatória também tem sido proposta, na qual a DSR é interpretada como sendo estado inflamatório regional prolongado que afeta a pele e as estruturas adjacentes, inclusive os ossos ${ }^{(3)}$.

\section{ABSTRACT}

The reflex sympathetic dystrophy (RSD) is a poorly understood, painful syndrome that consists of multiple clinical manifestations, including pain, swelling, dystrophic cutaneous changes and autonomic dysfunction involving one or more extremities. The RSD following herpes zoster is not always easily recognized, and only a few documented cases of this complication have been reported, what makes this report even more interesting. We present the case of a 65 years old white woman, with characteristic signs and symptoms of RSD in the right upper limb observed four weeks after she had had a typical herpes zoster involving the dermatomes. Early diagnosis and treatment with intranasal calcitonin and physiotherapy lead to progressive functional and pain improvements after one month.

Keywords: reflex sympathetic dystrophy, algoneurodystrophy, herpes zoster.

A causa da DSR é desconhecida. Muitos eventos precipitantes têm sido descritos, porém em 20\% dos casos nenhum fator causal foi observado. Trauma (incluindo cirurgias, fraturas, lesões com esmagamento tecidual e deslocamentos ou luxações articulares), no entanto, é a situação mais freqüentemente implicada. Outras condições, como injúrias por esforços repetitivos, lesões cerebrais isquêmicas ou expansivas, doenças coronarianas, hipertireoidismo, gravidez, diabetes mellitus, dislipidemias, infecções e fatores psicogênicos aumentam a susceptibilidade para o desenvolvimento dessa síndrome ${ }^{(1)}$.

A DSR como complicação do herpes zóster foi primeiramente descrita por Sudeck, em 1901(4), embora poucos

\footnotetext{
* Serviço de Reumatologia. do Hospital do Servidor Público Estadual de São Paulo "Francisco Morato de Oliveira" (HSPE/FMO). Recebido em 31/07/2003. Aprovado, após revisão, em 03/03/2004

1. Médico residente do Serviço de Reumatologia do HSPE/FMO.

2. Médico encarregado do ambulatório de Patologias da Coluna Vertebral e preceptor de ensino do Serviço de Reumatologia do HSPE/FMO.

3. Diretor do serviço de Reumatologia do HSPE/FMO.

Endereço para correspondência: Serviço de Reumatologia do Hospital do Servidor Público Estadual de São Paulo "Francisco Morato de Oliveira” (HSPE/FMO). R. Pedro de Toledo 1800, 9.andar, ala central, CEP 04039-004, São Paulo, SP. E-mail: cerir@nodel.com.br
} 
relatos da infecção pelo vírus varicela zóster, como causador desta síndrome, tenham sido publicados ${ }^{(5-11)}$.

Descrevemos a seguir o caso de uma paciente com características da DSR em membro superior direito que se iniciou aproximadamente 15 dias após o início de quadro clínico típico de herpes zóster.

\section{RELATO DE CASO}

Paciente de 65 anos, sexo feminino, branca, com antecedente de linfoma não-Hodgkin há cerca de 5 anos (realizada a última quimioterapia em 1999, com remissão completa), em controle ambulatorial no serviço de Hematologia do Hospital do Servidor Público Estadual de São Paulo "Francisco Morato de Oliveira". Apresentou, em dezembro de 2002, lesões cutâneas eritêmato-vesiculares, envolvendo região palmar direita e restante do membro superior direito, com trajeto dermatomérico, acompanhado de dor intensa. Procurou pronto-socorro, sendo avaliada pelo Serviço de Moléstias Infecciosas, recebendo diagnóstico de herpes zóster. Na ocasião foi internada e iniciado tratamento com aciclovir endovenoso $10 \mathrm{mg} / \mathrm{kg}$ a cada 8 horas, durante 6 dias e posteriormente complementado por via oral por mais 10 dias. Recebeu alta com diminuição das lesões herpéticas, que se apresentavam crostosas, e diminuição do quadro álgico.

Após alguns dias da resolução das lesões cutâneas, a paciente retorna em ambulatório, referindo dor de forte intensidade na mão direita, acompanhada de edema difuso, hiperemia e aumento da temperatura local (Figura 1). A mobilidade das articulações metacarpofalangeanas e interfalangeanas proximais estava limitada em virtude do edema e da dor. O ombro e o cotovelo direito não apresentavam alterações. A pele da mão direita, além das lesões residuais do herpes zóster, apresentava-se descamativa e hiperhidrótica. A radiografia simples de mãos e punhos realizada na ocasião mostrou rarefação óssea em regiões carpometacarpiana e interfalangeanas direitas (Figura 2).

A paciente recebeu tratamento com calcitonina nasal e fisioterapia motora, havendo melhora funcional e álgica importante após um mês de tratamento.

\section{DISCUSSÃO}

A infecção pelo vírus varicela zóster pode resultar em várias complicações neurológicas. As mais freqüentes são a neuralgia pós-herpética, neuropatia motora periférica, paralisia de nervo craniano, mielites, encefalites, vasculopatia trombótica cerebral, polirradiculite aguda e
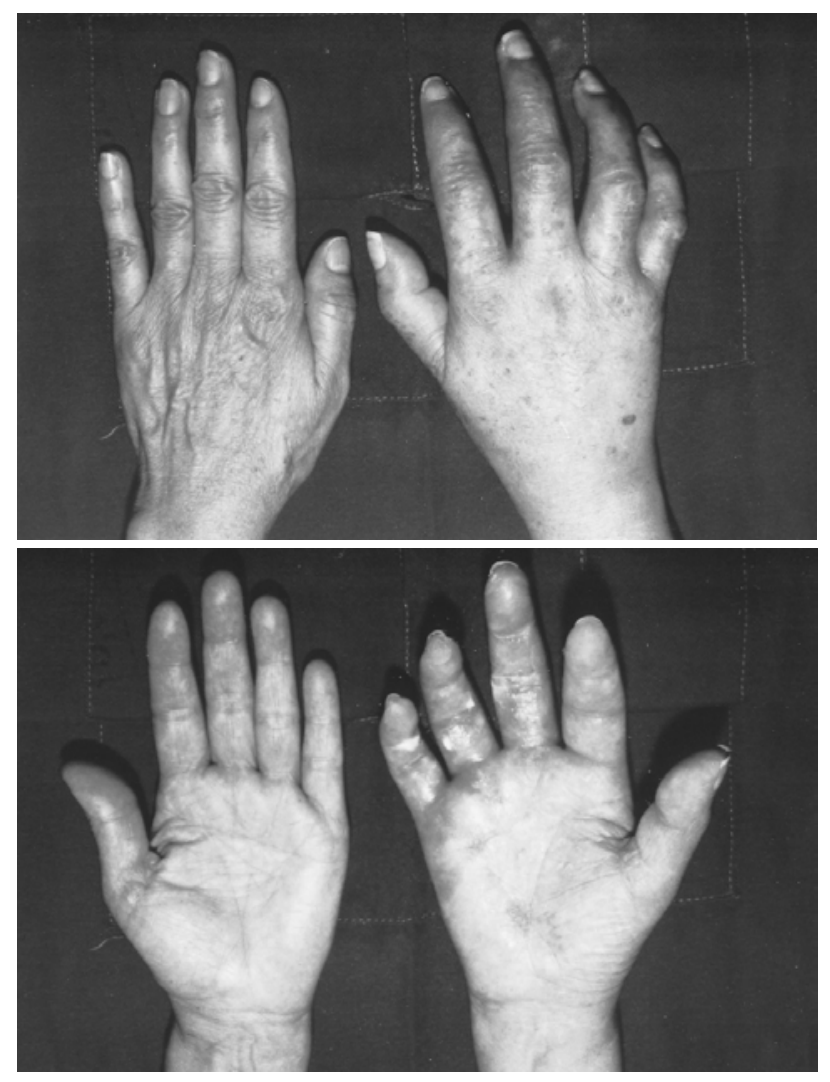

FigURA 1 - Mão direita mostrando edema difuso e lesões residuais do herpes zóster.

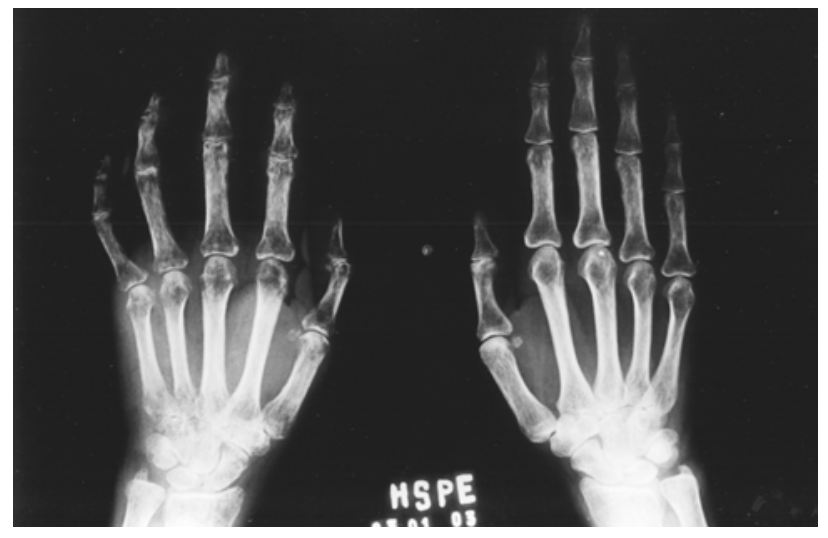

FiguRA 2 - Radiografias mostrando a rarefação óssea em regiões carpometacárpica e interfalangeanas direitas.

meningite asséptica ${ }^{(12,13)}$. A DSR é uma complicação rara e somente poucos relatos foram descritos na literatura ${ }^{(5-11)}$.

O herpes zóster provoca dor intensa, em virtude da neuralgia herpética e pós-herpética. Acredita-se que o estímulo nociceptivo aferente inicial possa sensibilizar 
múltiplos neurônios da cadeia de nervos simpáticos, resultando em uma resposta simpática excessiva. Devido a este estímulo e também pela própria infecção pelo varicela zóster, ocorre uma resposta inflamatória secundária, com conseqüente vasodilatação, edema e dor. Esta última, por sua vez, causa uma nova resposta simpática, estabelecendose um mecanismo amplificador. Além disso, a infecção pela varicela zóster pode alterar estruturas nervosas periféricas, levando a formação de sinapses anormais, usualmente entre nervos simpáticos eferentes e nervos sensitivos aferentes, ou nervos sensitivos periféricos, que são estimulados espontaneamente ${ }^{(1,2)}$.

A DSR é um processo dinâmico que pode progredir insidiosamente em três estágios $\left({ }^{14,16)}\right.$ :

Estágio I - Aparecimento de dor severa, aumento da sensibilidade da pele com o tato e pressão suave (hiperestesia), edema, espasmos musculares, rigidez e limitação de movimento. No início, a pele pode apresentar-se com aumento de temperatura, hiperemiada e ressecada, para depois evoluir com cianose, diminuição da temperatura e aumento da sudorese. Neste estágio, os casos mais leves podem regredir espontaneamente em algumas semanas ou responder rapidamente ao tratamento.

Estágio II - A dor torna-se mais severa e difusa, ocorre disseminação da inflamação e o edema pode estar endurado. A pele pode tornar-se grossa inicialmente, e na evolução apresentar-se delgada. A unha pode estar quebradiça e com sulcos proeminentes. Ocorre também atrofia muscular e osteopenia no local acometido.

Estágio III - Aparecimento de lesões cutâneas atróficas irreversíveis, além de dor de forte intensidade e deformidades estabelecidas.

\section{REFERÊNCIAS}

1. Kostin F: Reflex sympathetic dystrophy syndrome: a review. Clin Exp Rheumatol 10: 401-9 , 1992.

2. Roberts WJ: A hypothesis on the physiological basis for causalgia and related pain. Pain 24: 297-311, 1986.

3. Ecker A: Pathogenesis of reflex sympathetic dystrophy. Arch Neurol 46: 482, 1989

4. Sudek P: Uber die akute (reflecktorische) knochenatrophie nach entzundungen und verletzungen an den extremitaten und ihre Klinischen erscheinungen. Fortschr Geb Rontgenstr Nuklearmed Erganzungs 5: 277-93, 1901.

5. Ketz E, Schlizck H: Zoster in C5-C6 mit Sudeckscher Krankreit. Nervenartzt 39: 180-1, 1968.
Todas essas alterações apresentadas podem não estar presentes. Os sintomas descritos nos estágios I e II têm início no primeiro ano da doença. Alguns pacientes não progridem para o estágio III. Observa-se que alguns dos sintomas dos estágios I e II podem desaparecer espontaneamente, à medida que a doença progride para o estágio III.

O diagnóstico da DSR é baseado principalmente em dados clínicos (história e exame físico). Sempre é necessária a exclusão de outras enfermidades que trazem risco de vida ao paciente e que apresentam sintomatologia similar a DSR, como, por exemplo, a trombose venosa profunda. Não existem exames laboratoriais específicos para confirmação diagnóstica da DSR.

O tratamento inicia-se com a explicação da natureza da doença aos pacientes, informando os riscos e benefícios e custos diretos e indiretos das modalidades terapêuticas. A recuperação precoce da mobilidade e função da extremidade afetada é fundamental ${ }^{(15,17)}$. Um programa de reabilitação, em combinação com drogas ou técnicas analgésicas, aumenta sensivelmente a eficácia do tratamento. No serviço de Reumatologia do HSPE, utilizamos, nesses casos que apresentaram muita dor, a associação terapêutica, principalmente nas fases clínicas agudas e/ou subagudas, com a calcitonina. Esta, por sua vez, em doses de 200 UI, por spray nasal, geralmente duas vezes ao dia, durante três a quatro semanas, costuma diminuir acentuadamente a dor e o edema, por sua provável ação analgésica e de bloqueio da reabsorção óssea.

Embora a DSR seja uma condição com evolução clínica auto-limitada em grande parte dos casos, alguns pacientes podem evoluir por anos, tornando-se uma patologia permanente, levando a uma incapacidade funcional definitiva do membro afetado. O reconhecimento precoce desta entidade clínica é de suma importância na prática clínica diária.

6. Visitsunthorn U, Prete P: Reflex sympathetic dystrophy of the lower extremity: a complication of herpes zoster with dramatic response to propanolol. West J Med 135: 62-6, 1981.

7. Grosslight KR, Rowlingson JC, Boaden RW: Herpes zoster and reflex sympathetic dystrophy. Anesth Analg 65: 309-11, 1986.

8. Foster O, Askaria A, Lanham J, et al: Algoneurodystrophy following herpes zoster. Postgrad Med J 65: 478-80, 1989.

9. Chester MH: Segmental reflex sympathetic dystrophy involving the thumb: a rare complication of varicella - zoster infection. Anesthesiology 77: 1223-25, 1992.

10. Kishimoto N, Kato J, Suzuki T, et al: A case of RSD with complete disapperance of symptoms following intravenous ketamine infusion combined with stellate ganglion block and contínuos epidural block. Masui 44: 1680-84, 1995. 
11. Querol I, Cisneros T: Reflex sympathetic dystrophy syndrome following herpes zoster. Dermatol Gen Med 68: 179-82, 2001.

12. Elliot KJ: Other neurological complications of herpes zoster and their management . Ann Neurol 35: 57-61, 1994.

13. Flamholc L: Neurological complications in herpes zoster. Scans J Infect Dis 100(suppl): 35-40, 1996.

14. Dhar S, Klenerman L: Reflex sympathetic dystrophy (algodystrophy). Br J Rheumatol 32: 2-3, 1993.
15. Geertzen JHB, de Brujin $\mathrm{H}$, et al: Reflex sympathetic dystrophy: early treatment and psychological aspects. Arch Phys Med Rehabil 75: 442-6, 1994

16. Galer BS: Reflex sympathetic dystrophy syndrome. Lancet 334: 691, 1994.

17. Chard MD: Diagnosis and management of algoneurodystrophy. Ann Rheum Dis 50: 727-30, 1991. 